\title{
A Remark on the Moduli of the Roots of Algebraic Equations
}

\author{
Dragomir M. Simeunović
}

ABSTRACT. In this paper we obtain several upper bounds of the moduli of the roots of the algebraic equations.

The bounds of the moduli of the roots of the algebraic equations were investigated by many authors (see e.g. [1], [2], [3], [4]).

In this paper, for the algebraic equation

$$
z^{n}+a_{1} z^{n-1}+a_{2} z^{n-2}+\cdots+a_{n-1} z+a_{n}=0,
$$

the following theorems are proved. In the next let

$$
A_{k}=\left|a_{k}\right|, k=1,2, \ldots, n \text {. }
$$

Theorem 1. Let

$$
M_{1}=\max \left(A_{k}-A_{k-1}\right), \quad k=2,3, \ldots, n .
$$

Then the upper bound for the moduli of the roots of the equation (1) is

$$
1+A_{1}, \text { if } M_{1} \leq 0,
$$

$$
\frac{2+A_{1}+\sqrt{A_{1}^{2}+4 M_{1}}}{2} \text {, if } M_{1}>0 .
$$

Theorem 2. Let

$$
M_{2}=\max \left(A_{k}-A_{1}\right), k=2,3, \ldots, n
$$

and

$$
M_{3}=\max \left(\frac{A_{k}-A_{1}}{k-1}\right), k=2,3, \ldots, n .
$$

Then the upper bound for the moduli of the roots of the equation (1) is

$$
\begin{gathered}
1+A_{1}, \text { if } M_{2} \leq 0, \\
\frac{1+A_{1}+\sqrt{\left(1+A_{1}\right)^{2}+4 M_{2}}}{2}, \text { if } M_{2}>0
\end{gathered}
$$

2000 Mathematics Subject Classification. Primary: 12D10.

Key words and phrases. Roots of algebraic equations, Upper bounds for roots moduli. 
$\left(B_{5}\right)$

$$
1+A_{1}, \text { if } M_{3} \leq 0,
$$

$$
\frac{2+A_{1}+\sqrt{A_{1}^{2}+4 M_{3}}}{2} \text {, if } M_{3}>0 .
$$

Proof of Theorem 1. First, note that for $r>1$ we have the following relations

$$
\begin{gathered}
\frac{1}{r}+\frac{1}{r^{2}}+\cdots+\frac{1}{r^{n}}+\cdots=\frac{1}{r-1}, \\
\frac{1}{r}+\frac{2}{r^{2}}+\cdots+\frac{n}{r^{n}}+\cdots=\frac{r}{(r-1)^{2}}, \\
\frac{1}{r^{k}}=\frac{1}{(r-1) r^{k-1}}-\frac{1}{(r-1) r^{k}}, k=1,2, \ldots, n,
\end{gathered}
$$

which we shall use in the proof of the previous theorems.

Let $z=r e^{\theta i}(0 \leq \theta<2 \pi)$ the root of the equation (1), where

$$
|z|=r \text {. }
$$

Taking into account (2) and (9), from (1) we obtain the inequality

$$
1 \leq \frac{A_{1}}{r}+\frac{A_{2}}{r^{2}}+\cdots+\frac{A_{n-1}}{r^{n-1}}+\frac{A_{n}}{r^{n}} .
$$

In view of (8), the inequality (10) reduces to

$$
1 \leq \frac{A_{1}}{r-1}+\frac{1}{r-1}\left(\frac{A_{2}-A_{1}}{r}+\frac{A_{3}-A_{2}}{r^{2}}+\cdots+\frac{A_{n}-A_{n-1}}{r^{n-1}}\right)-\frac{A_{n}}{(r-1) r^{n}}
$$

wherefrom we obtain

$$
1<\frac{A_{1}}{r-1}+\frac{1}{r-1}\left(\frac{A_{2}-A_{1}}{r}+\frac{A_{3}-A_{2}}{r^{2}}+\cdots+\frac{A_{n}-A_{n-1}}{r^{n-1}}\right) .
$$

For $M_{1} \leq 0$, because of (3), (11) reduces to

$$
1<\frac{A_{1}}{r-1}
$$

wherefrom follows

$$
r<1+A_{1},
$$

which represents the proof of $\left(B_{1}\right)$.

For $M_{1}>0$, in view of (3) and (6), (11) reduces to

$$
\begin{aligned}
1 & <\frac{A_{1}}{r-1}+\frac{M_{1}}{r-1}\left(\frac{1}{r}+\frac{1}{r^{2}}+\cdots+\frac{1}{r^{n-1}}\right) \\
& <\frac{A_{1}}{r-1}+\frac{M_{1}}{r-1}\left(\frac{1}{r}+\frac{1}{r^{2}}+\cdots+\frac{1}{r^{n-1}}+\cdots\right)=\frac{A_{1}}{r-1}+\frac{M_{1}}{(r-1)^{2}},
\end{aligned}
$$

wherefrom follows that

$$
1<\frac{A_{1}}{r-1}+\frac{M_{1}}{(r-1)^{2}} .
$$


From (12) we obtain

$$
r<\frac{2+A_{1}+\sqrt{A_{1}^{2}+4 M_{1}}}{2}
$$

which represents the proof of $\left(B_{2}\right)$.

Proof of Theorem 2. Inequality (10) can be written in the form

$$
1 \leq \frac{A_{2}-A_{1}}{r^{2}}+\frac{A_{3}-A_{1}}{r_{3}}+\cdots+\frac{A_{n}-A_{1}}{r^{n}}+A_{1}\left(\frac{1}{r}+\frac{1}{r^{2}}+\cdots+\frac{1}{r^{n}}\right),
$$

wherefrom, because of (6) we have

$$
\begin{gathered}
1<\frac{1}{r}\left(\frac{A_{2}-A_{1}}{r}+\frac{A_{3}-A_{1}}{r^{2}}+\cdots+\frac{A_{n}-A_{1}}{r^{n-1}}\right) \\
+A_{1}\left(\frac{1}{r}+\frac{1}{r^{2}}+\cdots+\frac{1}{r^{n}}+\cdots\right) \\
=\frac{A_{1}}{r-1}+\frac{1}{r}\left(\frac{A_{2}-A_{1}}{r}+\frac{A_{3}-A_{1}}{r^{2}}+\cdots+\frac{A_{n}-A_{1}}{r^{n-1}}\right),
\end{gathered}
$$

which means that

$$
1<\frac{A_{1}}{r-1}+\frac{1}{r}\left(\frac{A_{2}-A_{1}}{r}+\frac{A_{3}-A_{1}}{r^{2}}+\cdots+\frac{A_{n}-A_{1}}{r^{n-1}}\right) .
$$

For $M_{2} \leq 0,(13)$ reduces to

$$
1<\frac{A_{1}}{r-1}
$$

wherefrom we obtain

$$
r<1+A_{1},
$$

which concludes the proof of $\left(B_{2}\right)$.

For $M_{2}>0$, because of (4) and (6), from inequality (13) we obtain

$$
\begin{aligned}
1 & <\frac{A_{1}}{r-1}+\frac{M_{2}}{r}\left(\frac{1}{r}+\frac{1}{r^{2}}+\cdots+\frac{1}{r^{n-1}}\right) \\
& <\frac{A_{1}}{r-1}+\frac{M_{2}}{r}\left(\frac{1}{r}+\frac{1}{r^{2}}+\cdots+\frac{1}{r^{n-1}}+\cdots\right)=\frac{A_{1}}{r-1}+\frac{M_{2}}{r(r-1)},
\end{aligned}
$$

that is

$$
1<\frac{A_{1}}{r-1}+\frac{M_{2}}{r(r-1)}
$$

From (14) we obtain

$$
r<\frac{1+A_{1}+\sqrt{\left(1+A_{1}\right)^{2}+4 M_{2}}}{2},
$$

which represents the proof of $\left(B_{4}\right)$. 
The inequality (13) can be written in the form

(15) $1<\frac{A_{1}}{r-1}+\frac{1}{r}\left(\frac{A_{2}-A_{1}}{1} \frac{1}{r}+\frac{A_{3}-A_{1}}{2} \frac{2}{r^{2}}+\cdots+\frac{A_{n}-A_{1}}{n-1} \frac{n-1}{r^{n-1}}\right)$.

For $M_{3} \leq 0$, from (15) we have

$$
1<\frac{A_{1}}{r-1}
$$

wherefrom we obtain

$$
r<1+A_{1},
$$

which represents the proof of $\left(B_{5}\right)$.

For $M_{3}>0$, because (5) and (7), (15) reduces to

$$
\begin{aligned}
1 & <\frac{A_{1}}{r-1}+\frac{M_{3}}{r}\left(\frac{1}{r}+\frac{2}{r^{2}}+\cdots+\frac{n-1}{r^{n-1}}\right) \\
& <\frac{A_{1}}{r-1}+\frac{M_{3}}{r}\left(\frac{1}{r}+\frac{2}{r^{2}}+\cdots+\frac{n-1}{r^{n-1}}+\cdots\right)=\frac{A_{1}}{r-1}+\frac{M_{3}}{(r-1)^{2}},
\end{aligned}
$$

that is

$$
1<\frac{A_{1}}{r-1}+\frac{M_{3}}{(r-1)^{2}}
$$

From (16) we obtain

$$
r<\frac{2+A_{1}+\sqrt{A_{1}^{2}+4 M_{3}}}{2}
$$

which represents the proof of $\left(B_{6}\right)$.

Theorem 3. Let

$$
M_{4}=\max \left(\frac{A_{k}-A_{k-1}}{k-1}\right), k=2,3, \ldots, n .
$$

Then the upper bound of the moduli of the roots of the equation (1) is

$$
1+A_{1}, \quad \text { if } M_{4} \leq 0,
$$

$$
s, \text { if } M_{4}>0,
$$

where $s>1$ is the root of the equation

$$
(r-1)^{3}-A_{1}(r-1)^{2}-M_{4}(r-1)-M_{4}=0 .
$$

Proof. Inequality (11) can be written in the form

$$
1<\frac{A_{1}}{r-1}+\frac{1}{r-1}\left(\frac{A_{2}-A_{1}}{1} \frac{1}{r}+\frac{A_{3}-A_{2}}{2} \frac{2}{r^{2}}+\cdots+\frac{A_{n}-A_{n-1}}{n-1} \frac{n-1}{r^{n-1}}\right) .
$$

For $M \leq 0$, from (19) we have

$$
1<\frac{A_{1}}{r-1}
$$


wherefrom we obtain

$$
r<1+A_{1}
$$

which represents the proof of $\left(B_{7}\right)$.

For $M_{4}>0$, because (17) and (7), (19) reduces to

$$
\begin{aligned}
1 & <\frac{A_{1}}{r-1}+\frac{M_{4}}{r-1}\left(\frac{1}{r}+\frac{2}{r^{2}}+\cdots+\frac{n-1}{r^{n-1}}\right) \\
& <\frac{A_{1}}{r-1}+\frac{M_{4}}{r-1}\left(\frac{1}{r}+\frac{2}{r^{2}}+\cdots+\frac{n-1}{r^{n-1}}+\cdots\right)=\frac{A_{1}}{r-1}+\frac{M_{4} r}{(r-1)^{3}},
\end{aligned}
$$

that is

$$
1<\frac{A_{1}}{r-1}+\frac{M_{4} r}{(r-1)^{3}} .
$$

From (20) we obtain

$$
(r-1)^{3}-A_{1}(r-1)^{2}-M_{4}(r-1)-M_{4}<0,
$$

wherefrom we conclude that root $s>1$ of the equation (18) is the upper bound of the moduli of the roots of the equation (1), which represents the proof of $\left(B_{8}\right)$.

\section{REFERENCES}

[1] M. Marden, Geometry of Polynomials, Amer. Math. Soc. Providence, R.I. 1966.

[2] S. Zervos, Aspects modernes de la localisation des séros des polynômes d'une variable, Ann. Sci. Ecole Norm. Sup., (3) 77 (1960), 303-410.

[3] P. Montel, Sur quelques limites pour les modules des séros des polynômes, Comment. Math. Helv., 7 (1934-35), 178-200.

[4] D. Marković, Granice korena algebarskih jednačina, Srpska Kraljevska Akademija, Glas CLXXXI, prvi razred 90, A. Matematičke nauke, Beograd 1939, 117-130.

Dragomir M. Simeunović

Mike Alasa 8

11000 BELGRADE

Serbia 